\title{
MODEL STUDY OF INHOMOGENEOUS LINE BROADENING IN EXCITONIC SPECTRA OF QUANTUM WELLS
}

\author{
G.V. Astakhov, V.P. KochereshKo, V.A. Kosobukin, D.R. Yakovlev
}

A.F. Ioffe Physico-Technical Institute, 194021, St. Petersburg, Russia

\author{
T. Wojtowicz, G. Karczewski, J. Kossut \\ Institute of Physics, Polish Academy of Sciences \\ Al. Lotników 32/46, 02-668 Warsaw, Poland
}

\section{W. Ossau and G. Landwehr}

Physikalisches Institut der Universität Würzburg, 97074, Würzburg, Germany

An approach is proposed to estimate separately parameters of homogeneous and inhomogeneous broadenings from an optical reflection line of a quasi-2D exciton. A phenomenological model is proposed to take into account statistically an inhomogeneous broadening of the exciton resonant spectra. The concept is applied to study a modulation-doped heterostructures with a single quantum well $\mathrm{CdTe} / \mathrm{CdMgTe}$. From exciton reflection lines taken in a magnetic field the temperature-dependent homogeneous and inhomogeneous broadening parameters as well as the exciton radiative decay rate are measured.

PACS numbers: $78.20 . \mathrm{Ci}, 78.66 .-\mathrm{w}$

\section{Introduction}

Optical reflection spectroscopy serves as a power tool for investigation and characterization of semiconductor nanostructures [1]. From excitonic spectra rich information can be obtained concerning resonant frequencies, radiative decay rates and homogeneous broadening of excitons as well as how those are affected by external fields. When an exciton state is inhomogeneously broadened, the spectral analysis becomes much more complicated and a possible accuracy of the obtained parameters is lowered. In structures with quantum wells (QWs) the effects of inhomogeneous broadening in excitonic spectra are of special importance because those are associated with unavoidable imperfections such as fluctuations of the thickness of a QW [2], in particular. However, despite a necessity in a study of inhomogeneously broadened exciton reflection spectra neither a concept nor systematic measurements are available until now. 
It is the aim of this paper to elaborate, both experimentally and theoretically, a procedure for analyzing homogeneous and inhomogeneous broadenings of exciton lines in reflection spectra. Basically, modulation spectroscopy ideas are employed below to separate out a contribution of inhomogeneous broadening in the linewidth.

\section{Basic concept and model structure}

We consider a heterostructure with a single quantum well (SQW) that is shown by the inset in Fig. 1. When the SQW interfaces are perfectly flat, the reflection coefficient at normal light incidence can be calculated as follows:

$$
R\left(\omega-\omega_{0}\right)=R_{\mathrm{b}}\left\{1+\frac{\Gamma_{0}\left[A\left(\omega_{0}-\omega\right)+B \gamma+C \Gamma_{0}\right]}{\left(\omega_{0}+\delta \omega_{0}-\omega\right)^{2}+\left(\gamma+\Gamma_{0}+\delta \Gamma_{0}\right)^{2}}\right\} .
$$

Here, $R_{\mathrm{b}}=[(n-1) /(n+1)]^{2}$ is the reflection coefficient of a background semiconductor whose relative refraction index is $n$, and the resonant term depending on the photon frequency $\omega$ is due to a quasi-2D exciton of the $\mathrm{QW}$. The parameters $R_{\mathrm{b}}, A, B$, and $C$ are defined by nothing but the surface of the structure. Next, $\omega_{0}, \gamma$, and $\Gamma_{0}$ are the exciton transition frequency, non-radiative and radiative decay rates for the SQW in an infinite semiconductor, while the corrections $\delta \omega_{0}$ and $\delta \Gamma_{0}$ are associated with interference field between the SQW and the sample surface.
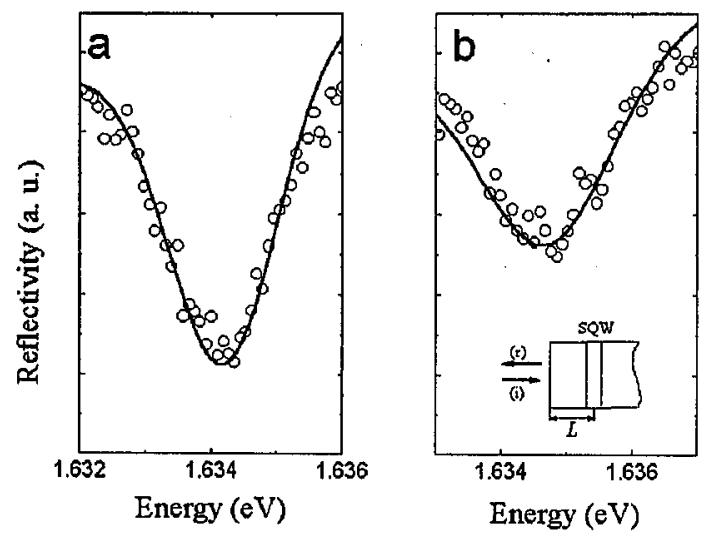

Fig. 1. Exciton reflectivity (in arbitrary units) in an applied magnetic field of $5.5 \mathrm{~T}$ and at a temperature of $6 \mathrm{~K}$ for $\sigma^{-}(\mathrm{a})$ and $\sigma^{+}(\mathrm{b})$ polarizations. Open circles correspond to experimental data, solid lines correspond to calculated results. The inset: scheme of the heterostructure, incident (i) and reflected (r) light waves are shown.

The nature of homogeneous and inhomogeneous broadenings is qualitatively different: the former is related to the exciton lifetime $\approx 1 / \gamma$, the latter is caused by a statistical distribution of the exciton transition frequency. In QWs an inhomogeneous broadening of exciton lines might originate from local fluctuations of $\mathrm{QW}$ thickness that unambiguously affects the exciton resonance energy through the 
carrier confinement. Considering a QW to consist regions (islands) of a constant thickness, our approach is based on the following ideas. First, when the lateral scale of these regions is larger than the exciton Bohr radius, the excitons inside can be considered as similar to those of infinite QW with the same thickness. And second, since a great number of such regions with random thicknesses are probed simultaneously in an optical experiment with a $\mathrm{QW}$, those should be treated statistically. In doing so, the averaged reflection coefficient

$$
\bar{R}\left(\omega-\bar{\omega}_{0}\right)=\int_{-\infty}^{\infty} \mathrm{d} \nu R(\omega-\nu) f\left(\nu-\bar{\omega}_{0}\right)
$$

is obtained after convolution of Eq. (1) with a normalized distribution function $f\left(\nu-\bar{\omega}_{0}\right)$ of the exciton transition frequencies.

Statistical properties of interfaces are unknown for a $\mathrm{QW}$. Therefore we use Gaussian statistics here with

$$
f\left(\nu-\bar{\omega}_{0}\right)=\frac{1}{\sqrt{2 \pi} \Delta} \exp \left(-\frac{\left(\nu-\bar{\omega}_{0}\right)}{2 \Delta^{2}}\right)
$$

that seems to be appropriate when many weak random scatterers independent of each other are probed, $\Delta$ being referred to as a parameter of inhomogeneous broadening. Besides, using measurements of excitonic spectra, the Gaussian correlation has been verified for grown semiconductor surfaces with random roughness [3]. After averaging (1) and (2) with the use of (3), one obtains

$$
\begin{aligned}
& \bar{R}\left(\omega-\bar{\omega}_{0}\right) \\
& \quad=R_{\mathrm{b}}\left\{1+\sqrt{\frac{\pi}{2}} \frac{\Gamma_{0}}{\Delta}\left[\frac{-A \delta \omega_{0}+B \gamma+C \Gamma_{0}}{\gamma+\Gamma_{0}+\delta \Gamma_{0}} \operatorname{Re} w(z)-A \operatorname{Im} w(z)\right]\right\} .
\end{aligned}
$$

Here, $w(z)$ is the probability integral of the complex argument [4]

$$
z=\frac{1}{\sqrt{2} \Delta}\left[\omega-\bar{\omega}_{0}+\mathrm{i}\left(\gamma+\Gamma_{0}+\delta \Gamma_{0}\right)\right] .
$$

It follows from Eq. (4) that an inhomogeneously broadened line contour is the simplest at $A=0$, where $A \approx \sin (2 n \omega L / c)$ with $L$ to be a distance between the SQW and the sample surface (Fig. 1). In this case, Eq. (4) describes Lorentzian, if $\Delta=0$, that is transformed into the Voigt contour, if $\Delta \neq 0$.

\section{Experiment}

As a model system we have studied $80 \AA$ thick $\mathrm{CdTe} / \mathrm{Cd}_{0.7} \mathrm{Mg}_{0.3} \mathrm{Te} \mathrm{QW}$ structures grown by molecular beam epitaxy technique on (100)-oriented GaAs substrates. The structures were modulation-doped with iodine in the barrier at a distance of $100 \AA$ from SQW. The concentration of quasi-2D electron gas in the SQW was of the order of $10^{11} \mathrm{~cm}^{-2}$. For our samples with $L=750 \AA$ and $n=2.64$ the above condition $A=0$ in Eq. (4) was well satisfied. The parameters $\omega_{0}, \Gamma_{0}+\delta \Gamma_{0}$, and $\gamma+\Gamma_{0}+\delta \Gamma_{0}$ were obtained separately from the location of a spectral peak, the peak intensity and its width, respectively. In order to estimate an additional parameter $\Delta$ that characterizes an inhomogeneous broadening we propose to modulate the line contour with an applied magnetic field through its influence on the excitonic polarization. 
The exciton reflection spectra of the above samples were measured in an applied magnetic field of $5.5 \mathrm{~T}$ in the Faraday geometry at the temperature of $6 \mathrm{~K}$. These spectra for the circular polarizations $\sigma^{-}$and $\sigma^{+}$are shown in Figs. 1a and $\mathrm{b}$, respectively. A small difference $(\approx 1 \mathrm{meV})$ between the resonant energies in the two polarizations is explained by the Zeeman splitting of the exciton level. The intensity and width of the exciton reflectivity contour are different for the polarizations $\sigma^{-}$and $\sigma^{+}$.

The presented excitonic spectra at $T=6 \mathrm{~K}$ were first approximated by Eq. (1) (or Eq. (4) with $\Delta=0$ ), the fitted parameters having been $\hbar \gamma^{-}=$ $1.05 \mathrm{meV}, \hbar \gamma^{+}=1.2 \mathrm{meV}, \hbar \Gamma_{0}^{-}=0.065 \mathrm{meV}$, and $\hbar \Gamma_{0}^{+}=0.052 \mathrm{meV}$, where the superscripts - and + correspond to $\sigma^{-}$and $\sigma^{+}$polarizations, respectively. The values of $\Gamma_{0}^{-}$and $\Gamma_{0}^{+}$differ by more than 20 per cent, that is essentially larger than an experimental error in measuring these parameters. On the other hand, the radiative decay rates $\Gamma_{0}^{-}$and $\Gamma_{0}^{+}$being defined by an overlapping of the electron and hole wave functions are to be independent of the angular momentum of the exciton. We believe this contradiction might be a consequence of neglecting an inhomogeneous broadening of the exciton line. In order to examine this issue, the above reflection spectra were fitted by Eq. (4) as well. In this case, the values $\hbar \gamma^{-}=0.33 \mathrm{meV}, \hbar \gamma^{+}=0.88 \mathrm{meV}$, and $\hbar \Gamma_{0}^{-}=\hbar \Gamma_{0}^{+}=0.052 \mathrm{meV}$ were obtained at $\hbar \Delta=0.71 \mathrm{meV}$, being the best fit value. As a result, the experimental and calculated reflection spectra show good agreement with each other for both $\sigma^{-}$ and $\sigma^{+}$polarizations at $T=6 \mathrm{~K}$ (Fig. 1).

\section{Discussion}

Having postulated existence of an inhomogeneous broadening, we have found experimentally the radiative decay rates $\hbar \Gamma_{0}^{-}=\hbar \Gamma_{0}^{+}=0.052 \mathrm{meV}$ to be constant in the temperature range 1.6 up to $15 \mathrm{~K}$. As presented in Fig. 2, the temperature dependences of the parameters $\Delta, \gamma^{-}$, and $\gamma^{+}$show that $\gamma^{-}<\gamma^{+}$at low temperatures, then $\gamma^{-}$increases with temperature while $\gamma^{+}$decreases. Such temperature behaviour of the homogeneous broadening parameters $\gamma^{-}$and $\gamma^{+}$can be ascribed to an exchange effect in exciton-electron scattering, when an electron from a photo-induced pair and one from quasi-2D electron gas replace each other [5]. In fact, at low temperatures an applied magnetic field orientates the spins of all equilibrium electrons along the field direction $(\uparrow)$. Let the polarization $\sigma^{-}$ of an incident photon induce the exciton angular momentum -1 that corresponds to the projections $(\uparrow)$ of electron spin and $(\Downarrow)$ of hole angular momentum on the direction of magnetic field. In this case, the above electron "exchange" should not modify the carriers wave function. Oppositely, the polarization $\sigma^{+}$induces an exciton angular momentum +1 with the projections $(\downarrow)$ and ( $\uparrow)$, respectively, that implies a change of the wave function after an "exchange" of electrons. As a result, the photoinduced electron is in optically inactive state $(\uparrow)(\Uparrow)$ whose angular momentum is +2 .

To summarize, the homogeneous linewidth $\gamma^{-}$for reflection of $\sigma^{-}$-polarized component should be smaller than $\gamma^{+}$of $\sigma^{+}$-polarized one, both parameters being of the form $\gamma^{\mp}=\tilde{\gamma}+\gamma_{\mathrm{ex}}^{\mp}$. Here, $\gamma_{\mathrm{ex}}^{\mp}$ describe contributions of exchange scattering that are considered to be proportional to the number of electrons whose spins are 


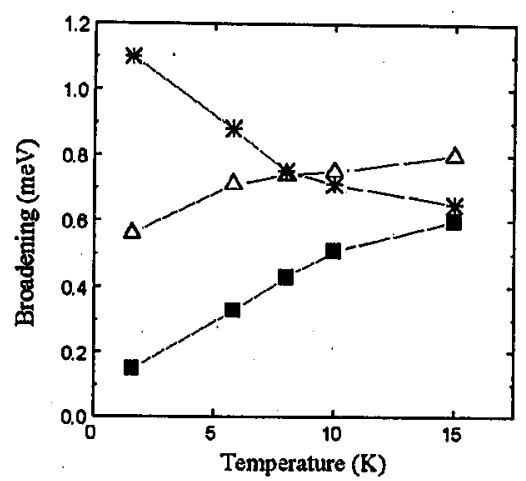

Fig. 2. Temperature dependences of the parameters: inhomogeneous broadening $\Delta$ (open triangles), and homogeneous broadening $\gamma^{-}$(solid squares) and $\gamma^{+}$(stars), the superscripts $\mp$ corresponding to the circular polarization $\sigma^{\mp}$.

$(\downarrow)$ or $(\uparrow)$, respectively, while $\tilde{\gamma}$ is ascribed to the other broadening mechanisms. At low temperature in magnetic field electrons occupy the lowest Zeeman level with spin ( $\uparrow)$. At higher temperatures the upper Zeeman level is occupied as well, and the number of electrons in the spin states $(\downarrow)$ increases. This is why the occupation numbers for electrons with spins $(\downarrow)$ and $(\uparrow)$ become close to each other with temperature and coincide above $T=20 \mathrm{~K}$, in which case, consequently, $\gamma^{-}=\gamma^{+}$.

\section{Conclusion}

We have proposed a systematic procedure for analysing an inhomogeneous broadening of exciton lines in optical spectra of quantum wells. The model under discussion verifies the separation of homogeneous and inhomogeneous contributions using a modulation of an exciton line in reflection spectrum by an applied magnetic field or, possibly, by temperature.

\section{Acknowledgments}

This work was supported by the RFBR grant No. 96-02-17929, RFBR-DFG grant No. 98-02-04089, the Deutsche Forschungsgemeinschaft (Os 98/5-1). The work in Poland was partially supported by the grant from Volkswagen Foundation.

\section{References}

[1] E.L. Ivchenko, P.S. Kop'ev, V.P. Kochereshko, I.N. Uraltsev, D.R. Yakovlev, S.V. Ivanov, B.Ya. Meltser, M.A. Kalitievskii, Sov. Phys. Semicond. 22, 495 (1988) [Fiz. Tekh. Poluprovodn. 22, 784 (1988)].

[2] J. Singh, K.K. Bajaj, S. Chaudhuri, Appl. Phys. Lett. 44, 805 (1984).

[3] V.A. Kosobukin, A.V. Sel'kin, Solid State Commun. 66, 313 (1988).

[4] Handbook of Mathematical Functions, Eds. M. Abramowitz, I.A. Stegun, Dover Publ. Inc., New York 1965.

[5] V.P. Kochereshko, D.R. Yakovlev, A.V. Platonov, W. Ossau, A. Waag, G. Landwehr, R.T. Cox, in: Proc. 23 Int. Conf. Physics of Semicond., Berlin 1996, Eds. M. Scheffler, R. Zimmermann, World Scientific, Singapore 1996, p. 1943. 